\title{
Cross-verification of the global gyrokinetic codes GENE and XGC
}

\author{
G. Merlo, ${ }^{1}$ J. Dominski, ${ }^{2}$ A. Bhattacharjee, ${ }^{2}$ C. S. \\ Chang, ${ }^{2}$ F. Jenko, ${ }^{1,3}$ S. Ku, ${ }^{2}$ E. Lanti, ${ }^{4}$ and S. Parker ${ }^{5}$ \\ ${ }^{1}$ The University of Texas at Austin, Austin, 78712 TX, USA \\ ${ }^{2}$ Princeton Plasma Physics Laboratory, Princeton, 08544 NJ, USA \\ ${ }^{3}$ Max Planck Institute for Plasma Physics, \\ Boltzmannstr. 2, D-85748 Garching, Germany \\ ${ }^{4}$ Ecole Polytechnique Fédérale de Lausanne (EPFL), \\ Swiss Plasma Center (SPC), CH-1015 Lausanne, Switzerland \\ ${ }^{5}$ University of Colorado, Boulder, 80309 CO, USA
}

\begin{abstract}
A detailed cross-verification between two global gyrokinetic codes, the core continuum code GENE and the edge particle-in-cell code XGC, for linear and nonlinear simulations of iontemperature-gradient modes is carried out. With recent developments in edge gyrokinetics, it may be feasible someday to describe the whole tokamak plasma on turbulence timescales using a coupled gyrokinetic simulation model. Before pursuing this, the core code (GENE) and the edge code (XGC) must be carefully benchmarked with each other. The present verification provides a solid basis for future code coupling research. Also included in the benchmarking is the global particle-in-cell code ORB5, to raise confidence in the quality of the obtained results. Excellent agreement between all three codes is obtained. Furthermore, in order to facilitate a benchmark framework for other codes, we make a specific effort to provide all relevant input parameters and precise details for each code.
\end{abstract}




\section{INTRODUCTION}

Turbulent transport in the core of tokamak plasmas is nowadays addressed on a daily basis with the help of gyrokinetic simulations [1, 2], using, e.g., the well-established core gyrokinetic code GENE [3, 4]. This includes numerous successful quantitative comparisons with experimental measurements [5-8]. Meanwhile, a key challenge in modeling tokamak transport is to create comprehensive gyrokinetic models of the edge, including the pedestal, with steep gradients, as well as the scrape-off layer, with field lines connecting to the divertor plates and the neutral particles recycled from the divertor plates. Significant progress in this area has been made with the development of the edge gyrokinetic code XGC [9-11].

As part of the Department of Energy Exascale Computing Project, the feasibility of coupling these two codes is being investigated. This is motivated by the fact that gyrokinetic codes are highly tuned for particular volumetric regions. A core gyrokinetic code like GENE is well optimized to model small amplitude, weak-gradient driven turbulence in the closed flux surfaces, whereas an edge gyrokinetic code like XGC operates best with large amplitude fluctuations and strong gradients across the magnetic separatrix and in the open magnetic field region in contact with material wall. Before such a core-edge coupled model can be realized, it is critical that the two codes are benchmarked within a reasonable level of accuracy. GENE and XGC solve the same gyrokinetic equations, so good agreement is expected when applied to the core plasma where both codes can solve the same problem. In practice, however, this may be difficult to achieve due to subtle differences in the discretization methods and the equilibrium models, for instance. It is important to pin down these differences prior to the development of a coupled model.

Although both GENE and XGC have been extensively and thoroughly benchmarked in various regimes (see, e.g., Refs. [9, 11-18]), there is not yet a direct comparison between these two codes. Moreover, previous benchmarks have used different sets of parameters, and thus even indirect comparisons are not possible. The present work addresses this gap by directly comparing the two global codes in toroidal geometry with conventional gyrokinetic benchmarking parameters. Beyond its value in view of future core-edge code coupling efforts, the results presented here also contribute to ongoing efforts on verification and validation of (global) gyrokinetic simulation models [14]. Also included in the benchmarking is the global Lagrangian particle-in-cell code ORB5 [19], thus providing more confidence in the quality 
of the obtained results. Furthermore, in order to facilitate the reproducibility of the same benchmark cases by other codes, we have made a specific effort to provide all relevant input parameters and precise details for each code.

The remainder of the paper is organized as follows. A brief description of the codes is given in Sec. II, while the description of the test case is outlined in Sec. III. Linear comparisons are presented in Sec. IV, and nonlinear results are described in Sec. V. Finally, conclusions are drawn in Sec. VI. Further details of the two codes, such the coordinate systems and the normalizations, are provided in the Appendices.

\section{THE CODES}

All three codes involved in this work - GENE, XGC, and ORB5 - solve for the time evolution of the gyro-center distribution function $F$ of each plasma species according to the gyrokinetic equation [20]

$$
\frac{\partial F}{\partial t}+\dot{\mathbf{X}} \cdot \nabla F+\dot{v}_{\|} \frac{\partial F}{\partial v_{\|}}=C
$$

where $\mathbf{X}$ is the gyro-center position in real space, $v_{\|}$is the velocity of the gyro-center parallel to the local equilibrium magnetic field $\mathbf{B}=B \mathbf{b}$, and $C$ accounts for the effects of collisions and sources, neglected in this work $(C=0)$. Note that even though all three codes can account for electromagnetic fluctuations, this work is limited to electrostatic perturbations. Therefore, all equations are given in this limit.

The equations of motion for a species with mass $m$ and charge $q$ read

$$
\begin{aligned}
& \dot{\mathbf{X}}=v_{\|} \mathbf{b}+\frac{B}{B_{\|}^{*}}\left(\mathbf{v}_{\nabla B}+\mathbf{v}_{\chi}+\mathbf{v}_{\kappa}\right) \\
& \dot{v}_{\|}=-\left(\frac{1}{m} \mathbf{b}+\frac{1}{m v_{\|}} \frac{B}{B_{\|}^{*}}\left(\mathbf{v}_{\nabla B}+\mathbf{v}_{\chi}+\mathbf{v}_{\kappa}\right)\right) \cdot(\mu \nabla B+q \nabla \tilde{\phi})
\end{aligned}
$$

where $\mathbf{v}_{\nabla B}=(\mu /(m \Omega B)) \mathbf{B} \times \nabla B$ is the grad-B drift velocity, $\mathbf{v}_{\chi}=\left(1 / B^{2}\right) \mathbf{B} \times \nabla \tilde{\phi}$ is the $\mathbf{E} \times \mathbf{B}$ velocity, and $\mathbf{v}_{\kappa}=\left(v_{\|}^{2} / \Omega\right)(\nabla \times \mathbf{b})_{\perp}$ is the curvature drift. Here, $\mu=\left(m v_{\perp}^{2}\right) /(2 B)$ stands for the guiding center magnetic moment, while $\tilde{\phi}$ is the gyroaverged electrostatic potential. Finally, the effective magnetic field $\mathbf{B}^{*}$ can be written as

$$
\begin{aligned}
\mathbf{B}^{*} & =\mathbf{B}+\frac{B}{\Omega} v_{\|} \nabla \times \mathbf{b} \\
& =B_{\|}^{*} \mathbf{b}+\frac{B}{\Omega} v_{\|}(\nabla \times \mathbf{b})_{\perp}=B_{\|}^{*} \mathbf{b}+\frac{B}{v_{\|}} \mathbf{v}_{\kappa} .
\end{aligned}
$$


The system of equations (1)-(3) is closed by the gyrokinetic Poisson law which, assuming a quasi-neutral background, reads

$$
\sum_{j} q_{j} \delta n_{j}=0
$$

where $\delta n$ is the perturbed density and the sum extends over all $j$ plasma species. $\delta n_{j}$ is evaluated as the zeroth order moment of the corresponding distribution function, resulting in two components: the so-called gyro-density and the polarization density [2], the latter resulting from the difference between guiding center and gyro-center coordinates, i.e. an effect related to finite amplitude field fluctuations. Equation (5) thus becomes in fact a linear integral equation for the electrostatic potential $\phi$ (see Eqs. (6)-(8)). The three codes use different numerical methods in order to solve Eqs. (1)-(3) and (5). They are briefly summarized in the following.

The GENE code $[3,4,21]$ is an Eulerian gyrokinetic code which solves Eq. (1) using the "method of lines" as follows. The distribution function is first discretized on a fixed grid in five-dimensional phase space and then numerically integrated. In order to exploit the strong anisotropy of fluctuations, GENE employs a field-aligned coordinate system $(x, y, z)$ to represent the fluctuation fields in configuration space. Here, $x$ defines the radial direction, $y$ the binormal direction, and $z$ parametrizes the position along a field line. The parallel velocity $v_{\|}$and magnetic moment $\mu$ serve as velocity space variables. The $y$ direction is Fourier transformed and, because of the tokamak axisymmetry, linear modes are characterized by a fixed $k_{y}$ value which is related to the toroidal mode number $n$ (see Appendix A). Finally, GENE makes use of the so-called $\delta f$ splitting technique, expressing the actual distribution as the sum of an equilibrium part $F_{0}$ (a local Maxwellian is assumed here) and a fluctuating component $f_{1}$, which is numerically evolved by the code.

Assuming an adiabatic response for the electrons, the quasineutrality equation solved by GENE reads

$$
\frac{2 \pi q_{i}}{m_{i}} \int B_{\|}^{*} \tilde{f}_{1 i} \mathrm{~d} v_{\|} \mathrm{d} \mu-\frac{q_{i}^{2} n_{0 i}}{T_{0 i}}\left[\phi-\int \frac{B}{T_{0 i}} \tilde{\tilde{\phi}} \exp \left(-\frac{\mu B}{T_{0 i}}\right) \mathrm{d} \mu\right]=\frac{e n_{0 e}}{T_{0 e}}\left(\phi-\langle\phi\rangle_{\mathrm{FS}}\right),
$$

where \langle\rangle$_{\mathrm{FS}}$ indicates a flux-surface average and $k_{\perp} \rho_{i}$ terms are retained to all orders in the evaluation of the ion polarization density term, $k_{\perp}$ being the wave number perpendicular to the magnetic field. 
The XGC code $[9,10,22]$ is a particle-in-cell (PIC) code which can simulate the whole plasma volume, from the magnetic axis to the scrape-off layer. Since the X-point and the magnetic axis are included in the calculation, magnetic coordinates cannot be used due to singularities there. XGC thus uses cylindrical coordinates $(R, \varphi, Z)$ to describe the particle motion, allowing for a general representation. In order to take advantage of the strong anisotropy of fluctuations, the mesh in real space follows the magnetic field-lines approximately and is structured as follows. All poloidal planes are equally spaced along the toroidal angle $\varphi$, and each of them is discretized in the same way using an unstructured triangular mesh that allows for the representation of complex diverted geometries and wall structures. The node points of the poloidal mesh follow the magnetic field lines.

$\mathrm{XGC}$ has different modes of operation, i) full-f: in this case the entire particle distribution function $f$ is represented with particle markers. ii) total-f: $f$ is split in three components, such that $f=f_{a}+f_{g}+f_{p}$. Here $f_{a}$ stands for the plasma background distribution function usually specified analytically, $f_{g}$ accounts for its slow evolution over local transport timescales (numerically represented on a grid), and $f_{p}$ represents a perturbation (represented with particle markers). The time evolution is evaluated according to a hybrid-Lagrangian $\delta f$ scheme, see Ref. [10]. iii) delta-f: the distribution function is split between a time invariant background and a perturbation, $f=f_{a}+f_{p}$, the latter described with markers and evolved using the weight evolution equation [23, 24]. Note that by initially setting $f_{g}=0$ and not transferring any weight from $f_{p}$ to $f_{g}$, the total-f scheme is in fact identical to the delta-f model. In the present work the delta-f model is used: $f_{a}$ is taken as a local Maxwellian and the grad-B drifts are ignored from the RHS of the weight equation, such that the numerical models of GENE and XGC are equivalent $\delta f$ models.

The electrostatic potential in XGC is solved with the quasi-neutrality equation in which the ion polarization term is evaluated using a Padé approximation, leading (in the adiabatic electron limit) to

$$
\nabla_{\perp} \frac{m_{i} n_{0}}{q_{i} B^{2}} \nabla_{\perp} \phi-\left(1-\nabla_{\perp} \rho_{i}^{2} \nabla_{\perp}\right) \frac{e n_{0}}{T_{0 e}}\left(\phi-\langle\phi\rangle_{\mathrm{FS}}\right)=\left(1-\nabla_{\perp} \rho_{i}^{2} \nabla_{\perp}\right) \delta \bar{n}_{i}
$$

where $\delta \bar{n}_{i}$ indicates the ion gyro-density.

The ORB5 code (a detailed description of the code can be found in Ref.[19]) is a $\delta f$ PIC code which solves the gyrokinetic system of equations in an axisymmetric system using 
magnetic coordinates. The ORB5 configuration space description is based on straight-fieldcoordinates $(s, \chi, \varphi)$ with $s=\sqrt{\psi / \psi_{\mathrm{LCFS}}}, \psi$ being the poloidal flux Eq. (A2) with $\psi$ defined to be zero at the magnetic axis, $\chi$ the straight-field-line poloidal angle (see Eq. (A3)) and $\varphi$ the toroidal angle. Similar to the GENE code, the distribution function of each species is split into an analytic background (a local Maxwellian is assumed in this work) and a perturbation, which is represented with markers. The motion of markers is described using the so-called $p_{\|}$-formalism, that is using the canonical parallel momentum $p_{\|}=m v_{\|}$(in the electrostatic limit) and the magnetic momentum $\mu$ as velocity space coordinates [25].

In ORB5, different expressions can be used for the ion polarization term appearing in the quasi-neutrality equation: (1) a long-wavelength approximation,

$$
\frac{q_{i}}{m_{i}} \nabla_{\perp} \cdot \frac{n_{0 i}}{\Omega_{0 i}^{2}} \nabla_{\perp} \phi-\frac{q_{e} n_{0 e}}{T_{0 e}}\left(\phi-\langle\phi\rangle_{\mathrm{FS}}\right)=\delta \bar{n}_{i}
$$

(2) a Padé approximant [26, 27], equivalent to the one used in XGC, and (3) an integral form, equivalent to the one used in GENE, which is exact to all orders [26].

\section{THE TEST CASE}

All benchmarks shown in present paper are carried out considering the realistic MHD magnetic geometry depicted in Figure 1. This equilibrium, defined as Case V in Ref. [28], is
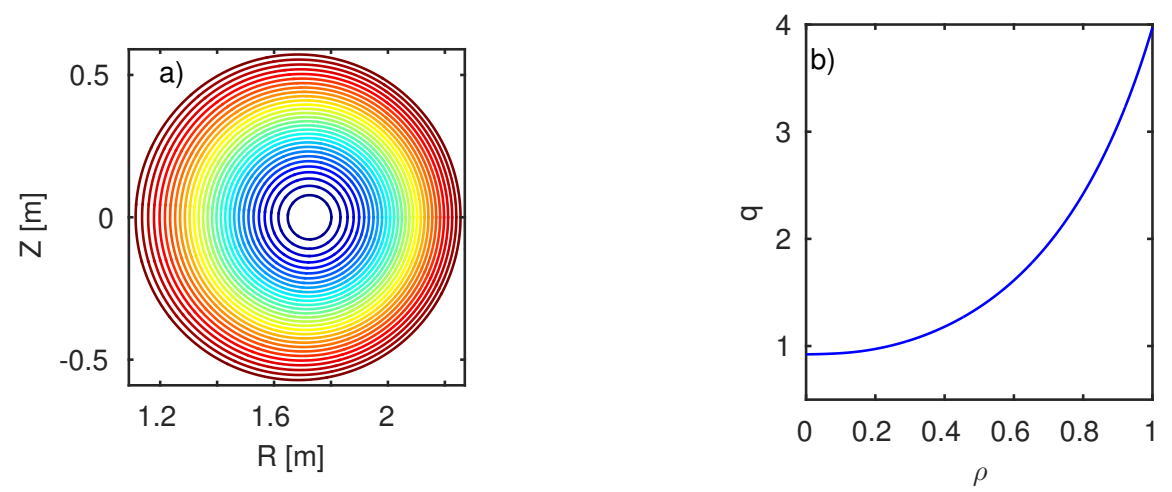

Figure 1: (Color online) a) MHD equilibrium considered for the benchmark, shown are constant contours of the poloidal magnetic flux function $\psi / \psi(\mathrm{LCFS})$, and (b) corresponding safety factor $q$ profile as a function of $\rho$.

based on the experimental DIII-D discharge underlying the Cyclone Base Case (CBC) [29], 
where the shape of the Last Closed Flux Surface (LCFS) has been modified from the experimental one to circular while attempting at keeping a safety factor profile $q$ (depicted in Figure 1) as close as possible to the original one. When reconstructing the equilibrium, nearly zero pressure is assumed such that the resulting flux-surfaces are approximated to be concentric (no Shafranov-shift). This magnetic geometry has already been used for carrying out both local and global benchmarks. [26, 30]

Throughout the entire paper, flux surfaces are labeled by the radial coordinate $\rho_{v o l}=$ $\sqrt{V / V_{\mathrm{LCFS}}}$, where $V$ is the volume enclosed by a given flux surface. One defines the geometrical minor radius as $r=\left[R_{\max }-R_{\min }\right] / 2, R_{\max }$ and $R_{\min }$ being, respectively, the maximum and minimum major radius of the flux surface evaluated at the elevation of the magnetic axis. The geometric center of the flux surface is given by $R_{\text {geom }}=\left[R_{\max }+R_{\min }\right] / 2$. The minor and major radius of the machine are therefore given by $a=r(\mathrm{LCFS})$ and $R_{0}=R_{\text {geom }}$ (LCFS). In this case $R_{0}=1.68 \mathrm{~m}$ and $a=0.59 \mathrm{~m}$ are assumed; the machine aspect ratio is $\epsilon=a / R_{0}=0.35$. A magnetic field on axis $B_{0}=2.09 \mathrm{~T}$ is considered.

A deuterium plasma with the same temperature profile for both ions and electrons is considered. Temperature and density profiles and their corresponding normalized logarithmic gradients are defined according to the following functional forms:

$$
\begin{aligned}
& \mathcal{A}\left(\rho_{\text {vol }}\right)=\mathcal{A}\left(\rho_{0}\right)\left[\frac{\cosh \left(\frac{\rho_{\text {vol }}-\rho_{0}+\delta \mathcal{A}}{\Delta \mathcal{A}}\right)}{\cosh \left(\frac{\rho_{v o l}-\rho_{0}-\delta \mathcal{A}}{\Delta \mathcal{A}}\right)}\right]^{-\kappa_{\mathcal{A}} \epsilon \Delta \mathcal{A} / 2} \\
& R_{0} / L_{\mathcal{A}}=\frac{\kappa_{\mathcal{A}}}{2}\left[\tanh \left(\frac{\rho_{\text {vol }}-\rho_{0}+\delta \mathcal{A}}{\Delta \mathcal{A}}\right)+\tanh \left(\frac{\rho_{v o l}+\rho_{0}+\delta \mathcal{A}}{\Delta \mathcal{A}}\right)\right],
\end{aligned}
$$

where $\mathcal{A}$ stands for both temperature $T$ and density $n . \mathcal{A}\left(\rho_{0}\right)$ indicates the corresponding reference values measured at $\rho_{0}=0.5$, which are chosen CBC-like, such that the associated finite size parameter $\rho^{*}=\rho_{i} / a$, the ratio between the ion gyro-radius and the plasma minor radius, assumes the value $\rho^{*}=1 / 180$. Finally, the maximum value of temperature (resp. density) logarithmic gradient is given by $\kappa_{T}$ (resp. $\kappa_{n}$ ).

The characteristic profile widths $\delta$ and $\Delta$ as well as $\kappa_{T}$ and $\kappa_{n}$ are chosen differently for linear and nonlinear comparisons, as will be detailed in the following sections. For completeness, the values of all relevant plasma parameters are also summarized in Table I. 


\begin{tabular}{lc}
\hline \hline$R_{0}[\mathrm{~m}]$ & 1.68 \\
$a[\mathrm{~m}]$ & 0.59 \\
$B[\mathrm{~T}]$ & 2.09 \\
$m_{\mathrm{D}}[\mathrm{kg}]$ & $3.3410^{-27}$ \\
$n_{0}=n(0.5)\left[10^{19} / \mathrm{m}^{3}\right]$ & 4.66 \\
$T_{0}=T(0.5)[\mathrm{keV}]$ & 2.25 \\
$\rho^{*}=\rho_{i} / a$ & $1 / 180$ \\
\hline \hline
\end{tabular}

Table I: Reference plasma parameters considered for the benchmark.

\section{LINEAR COMPARISONS}

In the first stage, a linear benchmarking is carried out, comparing growth rates and frequencies of the most unstable mode for different toroidal mode numbers $n$. In this context, it turns out to be helpful to use a sufficiently localized region of maximum gradient to avoid beating between different modes with similar growth rates from different radii. Thus, the profiles widths $\delta$ and $\Delta$ appearing in Eq. (9) are taken as 0.075 and 0.02 , respectively. CBClike gradients are considered, i.e., $\kappa_{T}=6.91$ and $\kappa_{n}=2.22$. The resulting profiles are shown in Figure 2.


Figure 2: (Color online) Radial profiles (solid lines) and corresponding normalized logarithmic gradients (dashed lines) of temperature (left) and density (right) used for the linear comparisons.

The GENE simulations described in this section have been performed considering a grid composed of $n_{x} \times n_{z} \times n_{v_{\|}} \times n_{\mu}=256 \times 32 \times 96 \times 32$ points, covering the radial domain $0.15 \leq \rho \leq 0.85$ (thus resulting in a grid spacing of $\sim 1.6 \mathrm{~mm}$ ) and the velocity space region 
$-4.6 \leq v_{\|} / v_{i} \leq 4.6,0 \leq \mu /\left(T_{0} B_{0}\right) \leq 10.8$, where $v_{i}=\sqrt{T_{0} / m_{\mathrm{D}}}$.

For the XGC simulation presented here, the poloidal unstructured grid has a characteristic length scale of $2-3 \mathrm{~mm}$. The number of triangle elements in each plane is $\sim 10^{6}$ for 2 mm grids, and $\sim 5 \times 10^{5}$ for $3 \mathrm{~mm}$ grids. The resolution of the toroidal grid is taken to be eight times the toroidal mode number $n$. For example, for $n=40$, one uses 320 toroidal points. The simulation domain extends from the magnetic axis to $\psi / \psi_{\mathrm{LCFS}}=0.9$. About $10^{7}$ marker particles are used, and for the gyro-averaging, a 32-point averaging technique is employed.

The ORB5 simulations discussed here were performed using $512 \times 1024 \times 512$ points in the radial, poloidal, and toroidal directions, respectively. The radial domain covers the entire plasma minor radius, discretized with a uniform grid. $10^{7}$ markers were used, while 16 points per gyro-orbit are employed in the gyro-averaging procedure.

The results obtained are shown in Figure 3, where the growth rate $\gamma$ and the real frequency $\omega_{r}$ obtained with the different codes are compared. Results are shown as a function of the toroidal mode number $n$, that can be uniquely defined for axisymmetric systems and independent from each code-specific coordinate system. Similarly, results are plotted in SI units to avoid the complication associated with different normalizations. To help the reader, the same color coding is used when comparing results: GENE results are shown in blue, $\mathrm{XGC}$ results in green, and ORB5 results in red. Frequencies and growth rates are also summarized in Table II.

As far as the linear growth rates are concerned, the three codes agree within $10 \%$ for all modes considered. Concerning the frequency, the curves agree within less than $3 \%$. We point out that the different codes use different approaches for solving the gyrokinetic Poisson equation. GENE and ORB5 currently implement an arbitrary-wavelength field solver, while XGC uses a Padé approximation. However, this is not found to significantly influence the growth rates for the toroidal mode numbers considered here.

The comparison between the codes is further pursued by inspecting the eigenfunction of the mode $n=24$. Figure 4 shows for each code a poloidal cross section of the electrostatic potential $\phi$ taken at the end of the simulation. A good qualitative agreement is observed, 

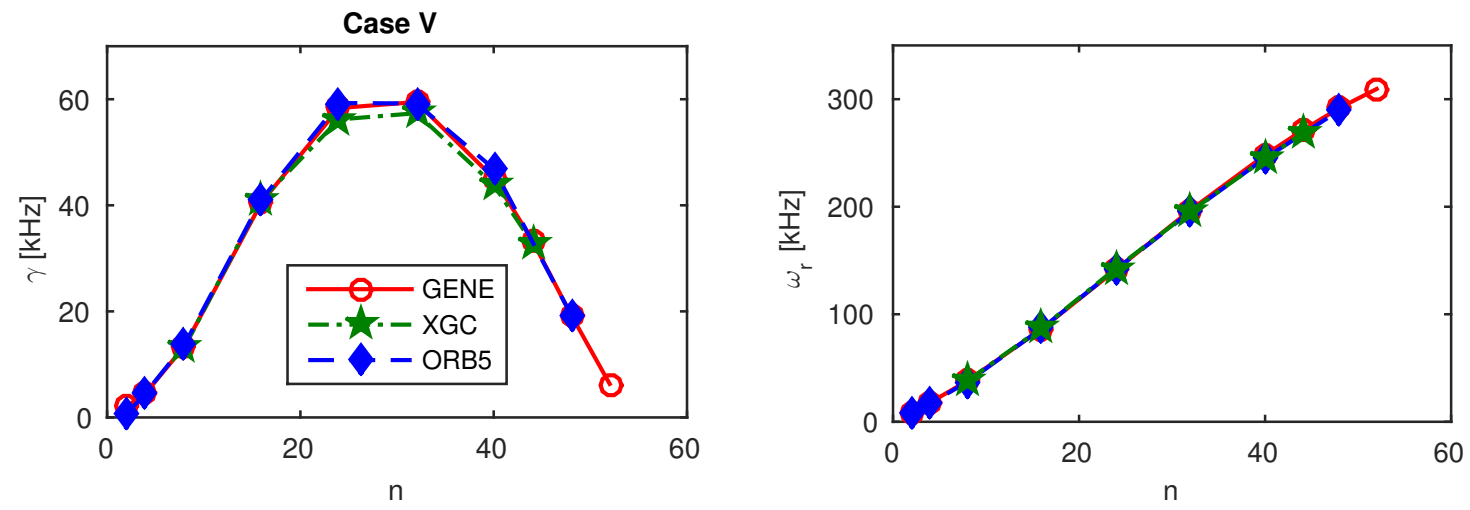

Figure 3: (Color online) Growth rates $\gamma$ (on the left) and real frequencies $\omega_{r}$ (on the right) for the most unstable mode as a function of the toroidal mode number $n$. GENE results are shown as red circles, XGC results as green stars, and ORB5 results as blue diamonds.

\begin{tabular}{lll}
\hline \hline $\mathrm{n}$ & $\gamma[\mathrm{kHz}]$ & $\omega_{r}[\mathrm{kHz}]$ \\
\hline \hline 2 & 2.03 & 8.45 \\
4 & 4.62 & 17.97 \\
8 & 13.41 & 38.03 \\
16 & 40.61 & 86.51 \\
24 & 58.35 & 141.62 \\
32 & 59.47 & 197.08 \\
40 & 45.14 & 248.08 \\
44 & 33.36 & 270.95 \\
48 & 19.37 & 291.83 \\
52 & 6.32 & 309.50 \\
\hline \hline
\end{tabular}

Table II: Frequencies and growth rates obtained with the GENE code, as shown in Figure 3.

with all codes reproducing similar eddy structures centered around the maximum logarithmic gradient region. These eigenfunction are then quantitatively compared in Figure 5. Subplot a) compares the mode amplitudes, averaged over the poloidal direction and re-normalized to their maximum value, while subplot $b$ ) shows the poloidal mode structure at $\rho_{v o l}=0.5$. In order to make this latter comparison, the eigenfunctions have been adapted such as to match the phase of the dominant poloidal mode ( $m=33$ for all codes). Even though some minor differences can be seen (e.g., a radial shift of the envelopes and small deviations in 

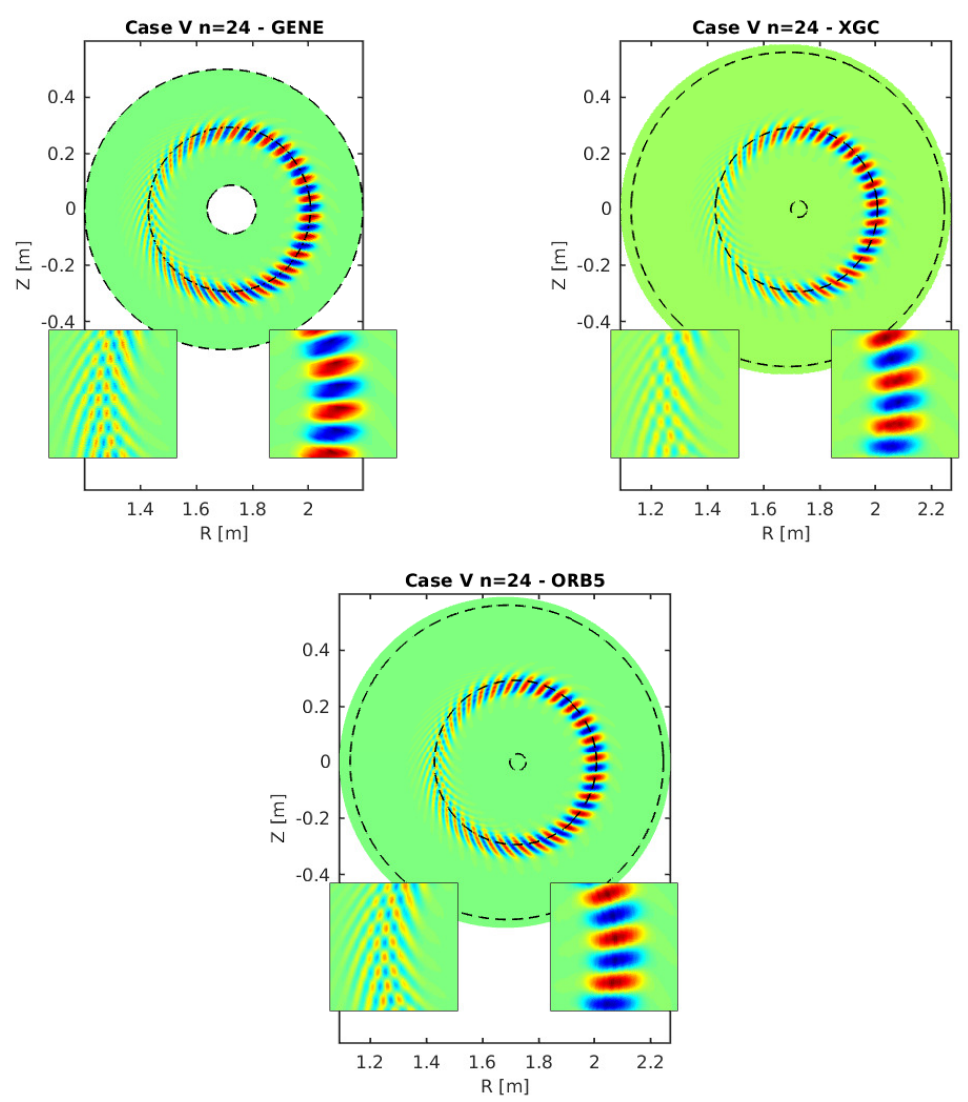

Figure 4: (Color online) Poloidal cross sections of the electrostatic potential associated with the $n=24$ mode obtained at the end of the simulation. Zooms on the inboard and outboard mid-planes are shown as well.
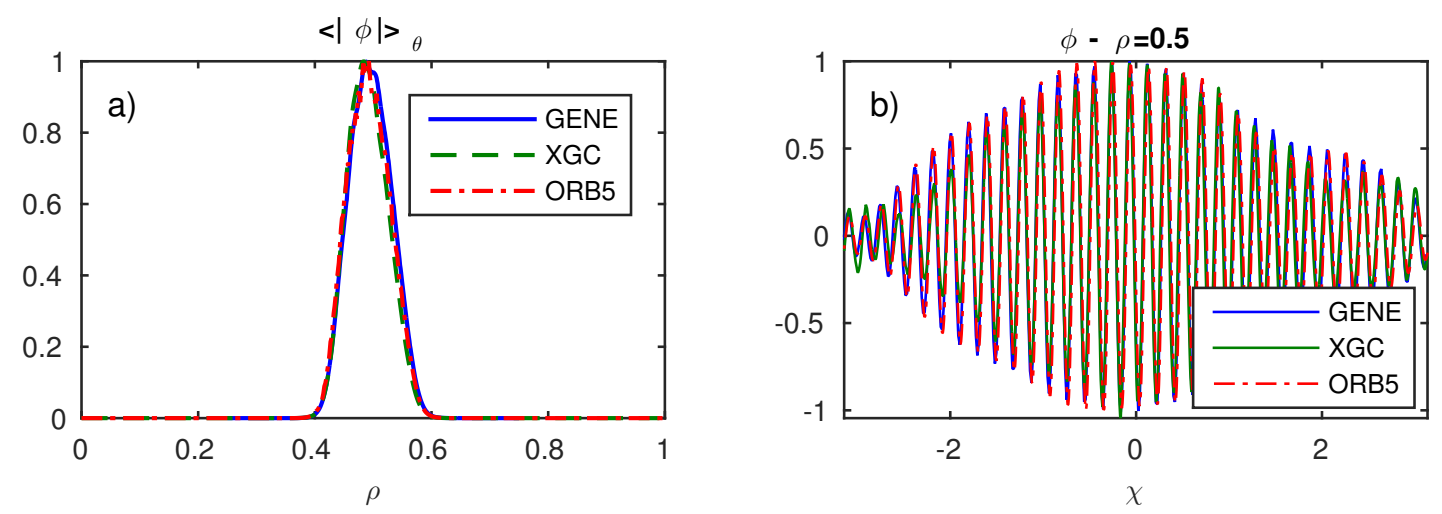

Figure 5: (Color online) Comparison of the eigenfunction associated with the mode $n=24(a)$ as a function of radius (poloidally averaged fluctuation) and (b) as a function of the straight-field-line poloidal angle $\chi$ at $\rho=0.5$.

the poloidal structure amplitude), the agreement can be considered excellent. 


\section{NONLINEAR COMPARISONS}

In a second step, a nonlinear verification is carried out. No sources are used in order to avoid the complications related to differences in the implementation of the source terms in the different codes. Multiple temperature profiles, characterized by different logarithmic gradient widths, are considered.

A specific effort is made to ensure that the codes use the same initialization conditions, which is a necessary prerequisite for being able to match the simulation results. Expressed in GENE coordinates, they read

$$
\hat{f}\left(x, k_{y}, z, v_{\|}, \mu\right)=A_{k_{y}} \exp \left[-\frac{1}{2}\left(\frac{x-0.5}{\sigma}\right)^{2}\right] \hat{F}_{\mathrm{M}}
$$

for all nonzero $k_{y}$ modes, $F_{\mathrm{M}}$ being the background local Maxwellian. Unless stated otherwise, $A_{k_{y}}=10^{-5}$ and $\sigma=10 \rho_{i}=a / 18$ are considered.

A first comparison is carried out considering flat gradient profiles with CBC values $\left(\kappa_{T}=6.91\right.$ and $\left.\kappa_{n}=2.22\right)$, extending over a major fraction of the plasma minor radius. Temperature and density profiles are computed according to Eq. (9), employing the same parameters as the ones used for the linear benchmark, with the exception of $\delta \mathcal{A}$, taken here as $\delta \mathcal{A}=0.35$. The corresponding profiles are shown in Figure 6. The main goal of
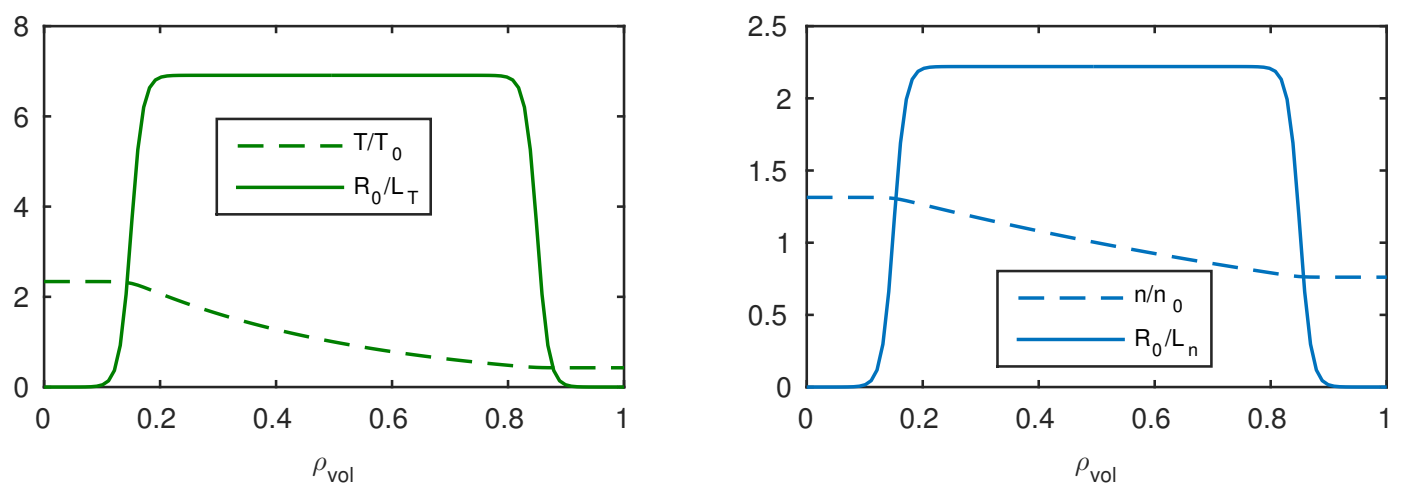

Figure 6: Radial profiles (solid lines) and corresponding normalized logarithmic gradients (dashed lines) of temperature (left) and density (right) profiles used for the nonlinear benchmark.

this choice is to have a realistic profile, avoiding at the same time strong finite machine size effects due to the small value of $\rho_{\mathrm{eff}}^{*}$. Here, $\rho_{\mathrm{eff}}^{*}=\rho_{i} / \delta \mathcal{A}$, which is the ratio between the ion Larmor radius and the characteristic radial profile width, provides a measure of the 
effective finite machine size effects [31].

All simulations are carried out considering a toroidal wedge corresponding to a third of the tokamak $\left(n_{\min }=3\right.$ being the minimum toroidal mode number considered) and using 32 toroidal modes. The remaining directions are discretized as follows.

The GENE simulations consider the radial annulus domain $0.05 \leq \rho_{\text {vol }} \leq 0.95$ and the velocity space region $-4.6 \leq v_{\|} \leq 4.6,0 \leq \mu /\left(T_{0} B_{0}\right) \leq 21$. The runs are performed with a grid of $n_{x} \times n_{k_{y}} \times n_{z} \times n_{v_{\|}} \times n_{\mu}=176 \times 32 \times 24 \times 76 \times 45$ points.

The XGC simulations are performed on a spatial grid composed of $n_{R Z} \times n_{\varphi}=232011 \times 64$ points; here, $n_{R Z}$ represents the number of grid points in a poloidal plane, while $n_{\varphi}$ is the number of the poloidal plane in the toroidal direction. The unstructured poloidal grid has a characteristic length of about $3 \mathrm{~mm}$. The simulation domain covers the radial region $\rho_{v o l} \leq 0.95$ and uses 938 million marker particles. For gyro-averaging, a 32-point averaging technique is used.

The ORB5 runs are carried out on a grid of $n_{s} \times n_{\chi} \times n_{\varphi}=256 \times 1024 \times 512$ points, $(s, \chi, \varphi)$ representing the radial, poloidal, and toroidal directions. The simulation domain covers the entire plasma minor radius. 500 million markers are used, and a 16-point averaging technique is employed for the gyro-averaging. In order to reduced the computational cost of the runs, the long-wavelength approximation in the field solver is used.

In Figure 7, we plot the simulated heat flux per particle, $Q_{i} / n_{i}$, in $\mathrm{J} / \mathrm{ms}$ as a function of the radial coordinate and time. Here, $Q_{i}=\left\langle\mathbf{Q}_{\mathbf{i}} \cdot \nabla \rho_{v o l} /\left|\nabla \rho_{v o l}\right|\right\rangle$ is the ion heat flux, with $\mathbf{Q}_{\mathbf{i}}=\int\left(m v^{2} / 2\right) f \mathbf{v}_{\chi} \mathrm{d}^{3} v$, where $f$ is the fluctuating part of the distribution function and $\mathbf{v}_{\chi}$ is the $\mathbf{E} \times \mathbf{B}$ velocity. $\langle\cdot\rangle$ indicates a flux-surface average. The corresponding evolution of the logarithmic temperature gradient is shown in Figure 8.

As expected, the three codes exhibit a similar behavior, with an initial exponential growth phase of the microinstabilities, followed by a nonlinear saturation phase. Since no sources are used, the turbulent transport leads to a flattening of the driving gradients, eventually shutting off the turbulence. Minor differences can be observed, in particular during the nonlinear phase of the simulations. Such differences, due to the turbulent nature of the fluctuations and the different numerical discretization techniques used by the different codes, are expected and unavoidable. From a statistical point of view, which deduces the meaningful macroscopic quantities, however, the agreement between the codes is very good. This 


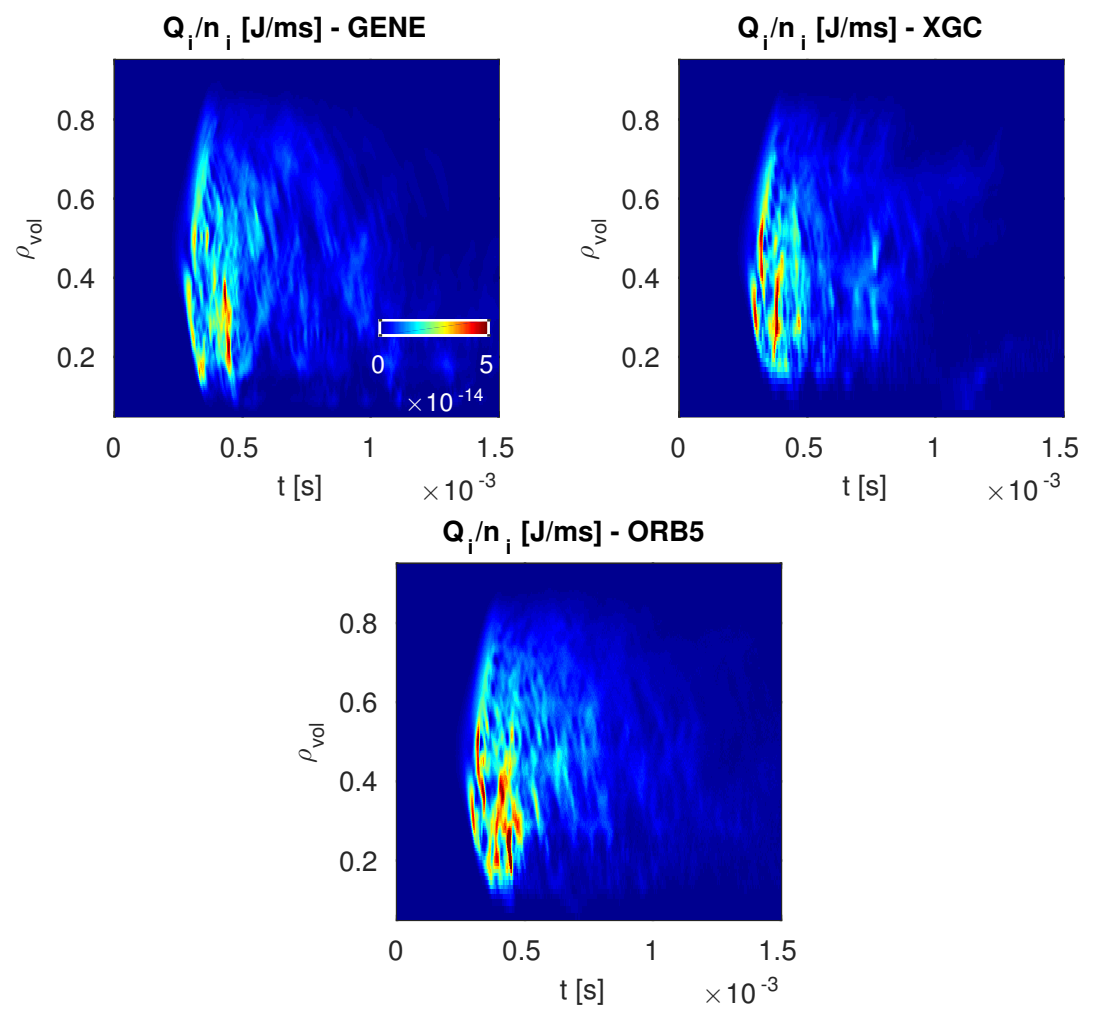

Figure 7: (Color online) Simulated heat flux per particle in $\mathrm{J} / \mathrm{ms}$ as a function of the radial coordinate $\rho_{v o l}$ and time $t$. Shown, from the left to the right, are the results obtained with GENE, XGC, and ORB5. The same color scale is used for the three plots. [Associated datasets available at http://dx.doi.org/10.5281/zenodo.898212] (Ref. [32])

is demonstrated in Figures 9 and 10, where the fluxes and gradients are compared quantitatively. Here, in order to obtain better statistics, we plot radially averaged quantities, considering the three radial windows $[0.2-0.8],[0.3-0.7]$, and $[0.4-0.6]$.

We would like to point out the excellent agreement obtained in the initial linear phase $\left(t<2 \cdot 10^{-4} \mathrm{~s}\right)$ where, thanks to having used the same initial condition, the time traces obtained with the different codes are on top of each other without any time delay. This is true for both fluxes and gradients, and independently from the radial window considered for averaging. A similar level of agreement is obtained when entering the saturation phase $\left(3 \cdot 10^{-4} \mathrm{~s}<t<4 \cdot 10^{-4} \mathrm{~s}\right)$ and the initial decay phase of the turbulence. Somewhat greater fluctuations in ORB5 can be attributed to the long-wave length approximation used in the exercise for computational time-saving. As already mentioned, deviations between the codes are observed due to the different numerical schemes employed and the chaotic 

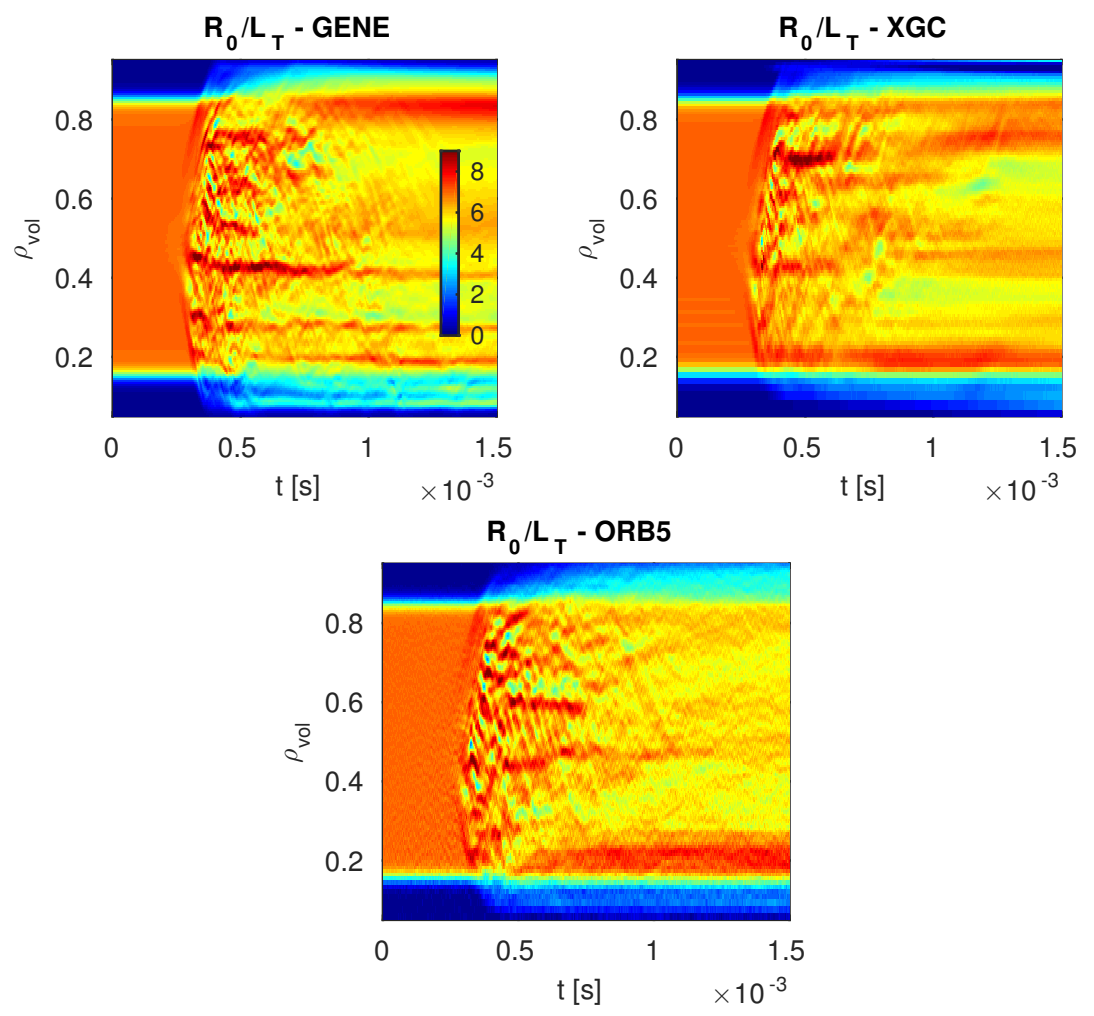

Figure 8: (Color online) Same as Figure 7, but showing the evolution of the logarithmic gradient $R_{0} / L_{T}$. [Associated datasets available at http://dx.doi.org/10.5281/zenodo.898212] (Ref. [32])

nature of turbulence. However, they are small and do not significantly influence the final value of the logarithmic temperature gradient. Greater difference in $R_{0} / L_{T}$ at the final stage $\left(t>0.7 \cdot 10^{-3} s\right.$ between XGC and GENE is not very meaningful because the turbulent fluctuation is already weak at the time due to the relaxed gradient and further relaxation is coming from non-turbulent effects unique to the discretization techniques.

The relaxed logarithmic gradient profile, time averaged from $t=10^{-3} \mathrm{~s}$, is shown in Figure 11. Differences between XGC and GENE codes are below 5\%, which can be considered quite satisfactory.

Finally, in order to gain further confidence in the code agreement, different widths of the backgrounds logarithmic gradient profile are considered. For this purpose, two other profiles are used, and the code results are confronted. The results of these additional comparisons are displayed in Figures 12 and 13. For simplicity, we only show the comparison between the fluxes and logarithmic gradients averaged over the interval [0.4-0.6], as well as the final 


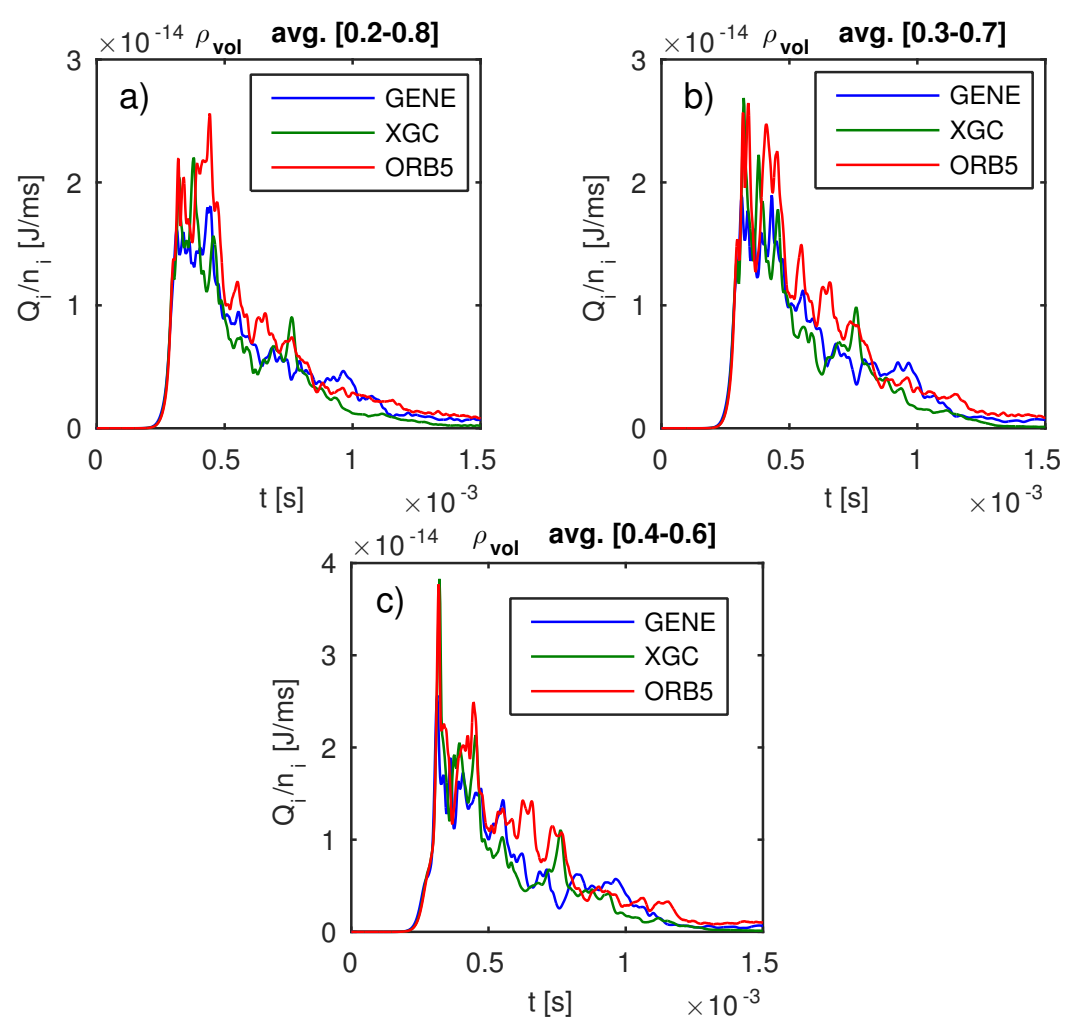

Figure 9: Time evolution of the ion heat flux per particle, radially averaged over the intervals (a) $0.2 \leq \rho_{v o l} \leq 0.8$, (b) $0.3 \leq \rho_{\text {vol }} \leq 0.7$, and (c) $0.4 \leq \rho_{\text {vol }} \leq 0.6$. Shown in blue, green, and red are the results obtained with GENE, XGC, and ORB5, respectively.

temperature profile. Very good agreement is once again obtained for all considered profiles.

\section{CONCLUSIONS}

A series of successful benchmarks has been carried out between the two state-of-theart global gyrokinetic codes GENE and XGC. The main motivation of this work is their foreseen coupling (treating, respectively, the core and edge region of a fusion plasma) in order to build a model spanning the whole plasma volume (from the magnetic axis to the material boundary), an effort carried out within the framework of the Exascale Computing Project. Moreover, it represents the most detailed comparison between XGC and any other gyrokinetic code to date.

To this aim, GENE and XGC have been directly confronted for the first time. This verification study also involved the ORB5 code, which provided a third set of independent 

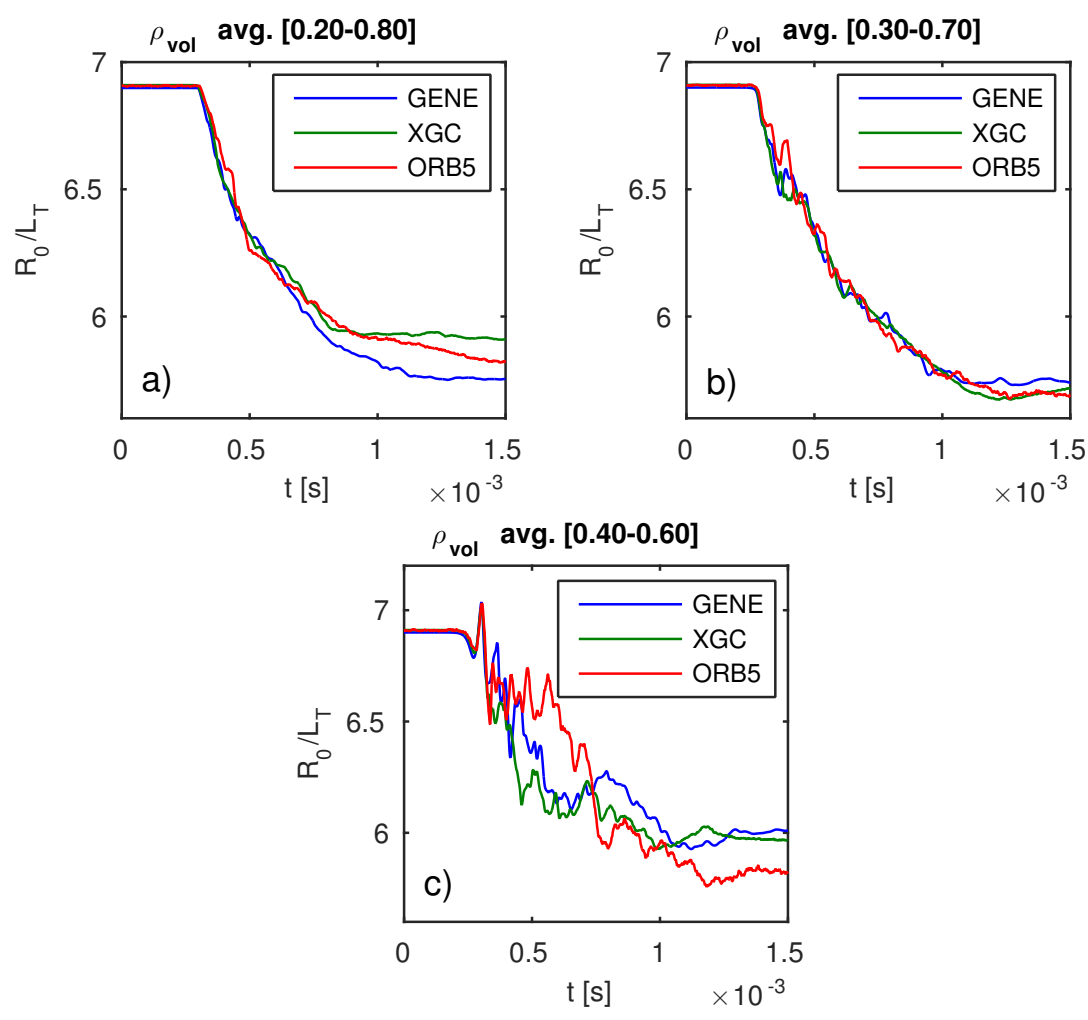

Figure 10: Same as Figure 9 but showing the evolution of the logarithmic temperature gradient $R_{0} / L_{T}$.

results to further strengthen the confidence in our results. Additionally, a deliberate effort was made to provide all relevant benchmarking details, so that any other code in the fusion community can carry out the same tests and reproduce our results. This includes making publicly available each codes' outputs [32]. For the sake of completeness, GENE input files are made available as well.

For this verification study, we have considered the simplest but still meaningful scenario, namely collisionless ion-temperature-gradient modes with an adiabatic electron response. Linear comparisons were carried out, recovering in all cases a very good agreement with respect to frequencies, growth rates, and mode structures of the most unstable mode for any given toroidal mode number. Nonlinear benchmarks were also performed, neglecting for simplicity - heat sources. The codes agree regarding the spatial and temporal evolution of the simulated heat flux as well as of the temperature profile, which exhibits a relaxation towards similar values. Such a good agreement is obtained for different choices of background profiles. 


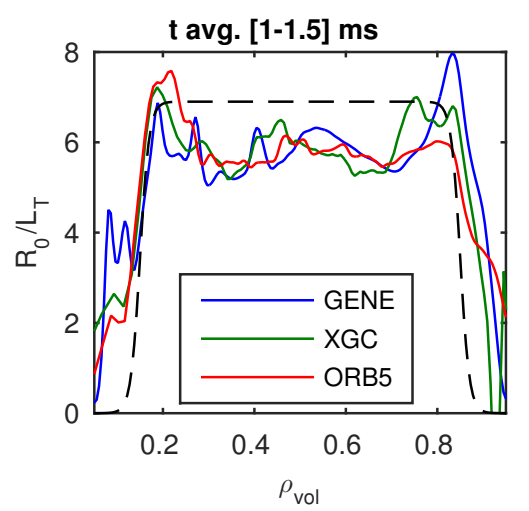

Figure 11: Relaxed logarithmic gradient profile, time averaged from $\mathrm{t}=10^{-3} \mathrm{~s}$. The initial background profile is indicated with a dashed black line. Shown in blue, green, and red are the results obtained with GENE, XGC, and ORB5, respectively.

This work constitutes the basis on which GENE and XGC will be coupled in order to build the first core-edge coupled gyrokinetic simulation model capable of modeling the entire plasma volume from magnetic axis to material boundary. The comparisons described in this paper are only a first step. The present verification study will be extended in the future, adopting a more complex and realistic physical model, e.g., considering a gyrokinetic response for electrons, adding heat sources, and retaining the effect of collisions.

\section{SUPPLEMENTARY MATERIAL}

See supplementary material for a description of the normalizations adopted by each code.

\section{ACKNOWLEDGEMENTS}

Useful discussions with A. B. Navarro, T. Görler, S. Janhunen and B. McMillan are greatfully acknowledged. This work was supported by the U.S. Department of Energy under the Exascale Computing Project (17-SC-20-SC). Part of this work was supported by a grant from the Swiss National Supercomputing Center (CSCS) under project IDs s704 and s513. This research used resources of the Oak Ridge Leadership Computing Facility at the Oak Ridge National Laboratory, which is supported by the Office of Science of the U.S. Department of Energy under Contracts Nos. DE-AC05-00OR22725. and DE-AC02$05 \mathrm{CH} 11231$. 

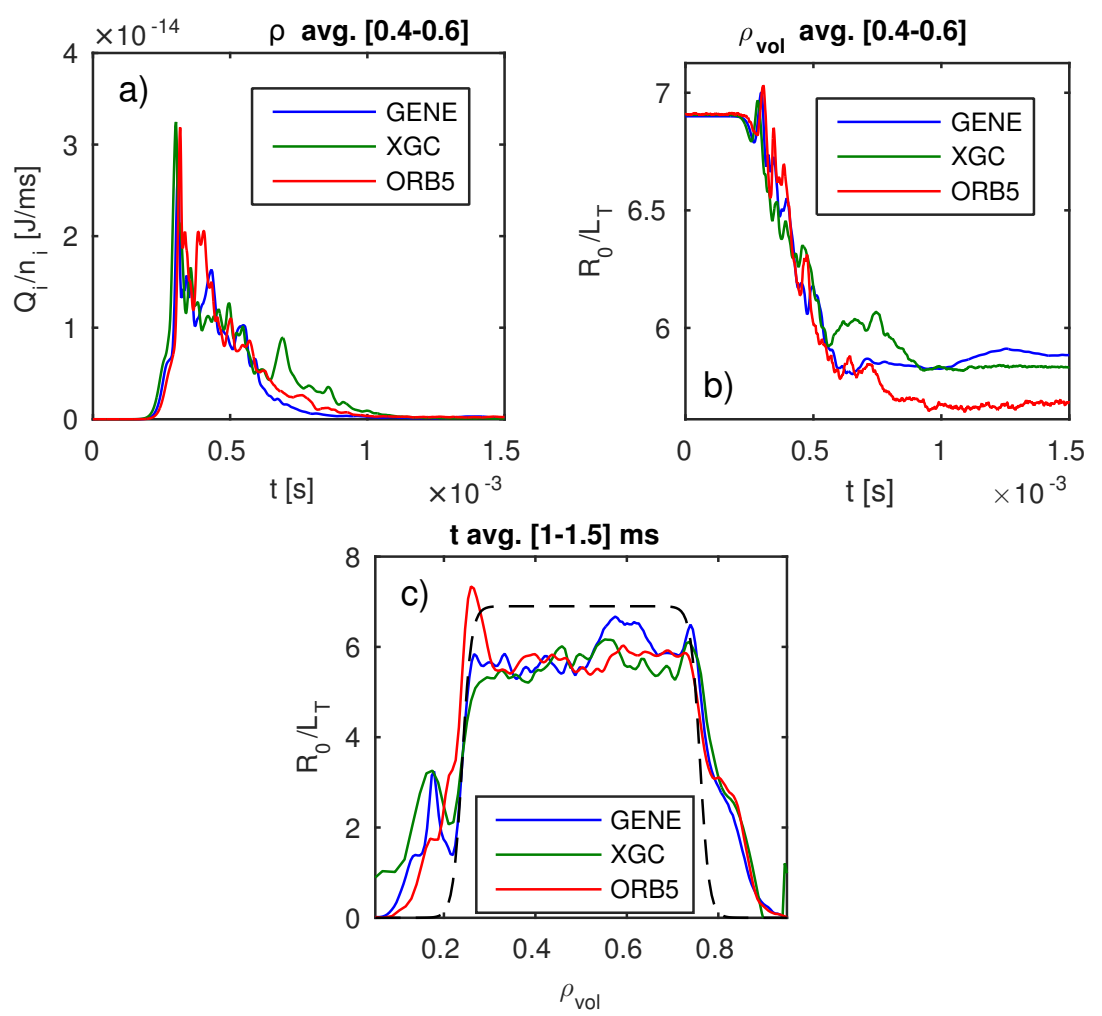

Figure 12: Benchmark considering $\Delta \mathcal{A}=0.26$ in Eq. (9). Shown, from the left to the right, are the time trace of the heat flux (radially averaged over $0.4 \leq \rho_{v o l} \leq 0.6$ ), the time trace of the logarithmic gradient, and the relaxed radial profile of $R_{0} / L_{T}$. GENE results are shown in blue, XGC results in green, and ORB5 results in red. 

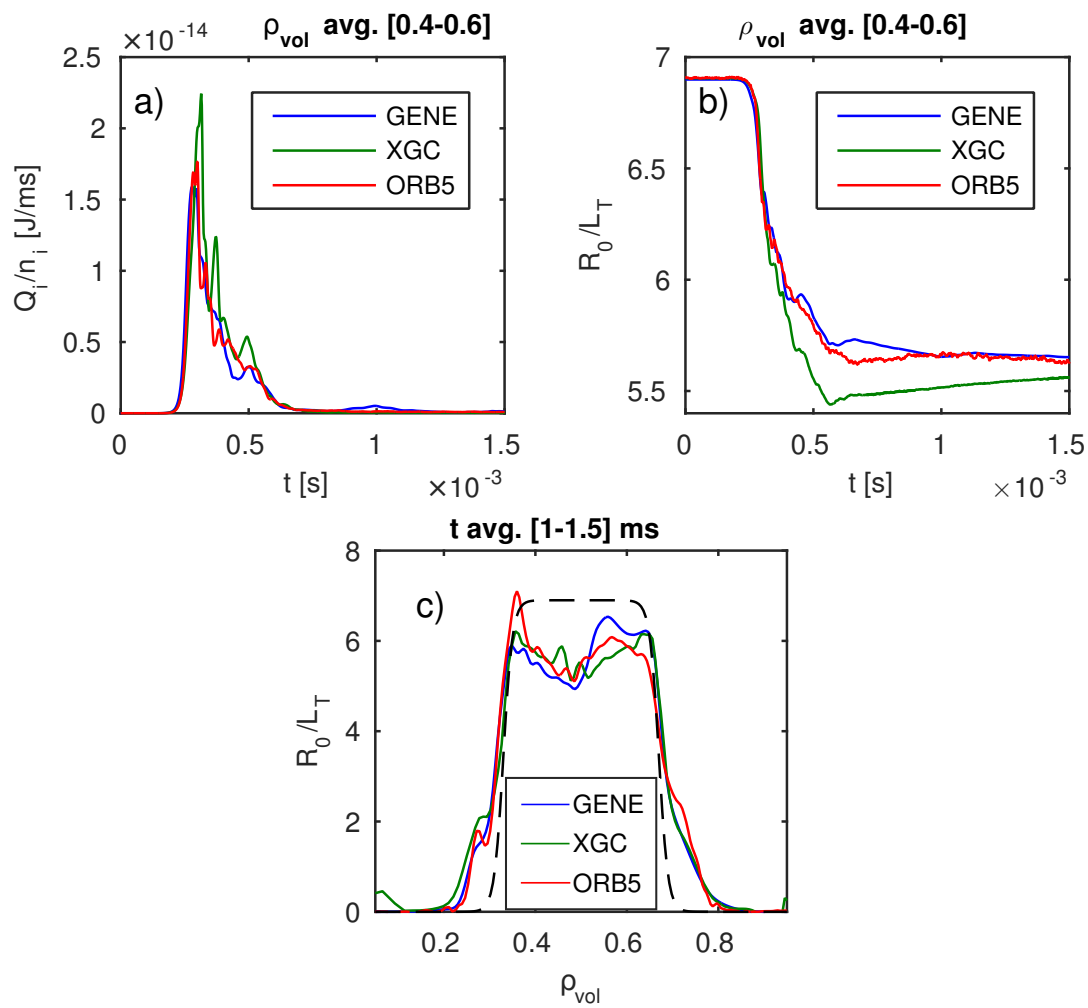

Figure 13: Same as Figure 12, but showing results obtained with $\Delta \mathcal{A}=0.17$. 


\section{Appendix A: The GENE coordinate system}

In order to discretize configuration space, GENE uses a field-aligned coordinate system $(x, y, z)$, expressed in terms of the magnetic straight field line coordinate system $(\psi, \chi, \varphi)$ as follows:

$$
\left\{\begin{array}{l}
x-x_{0}=C_{x}(\psi), \\
y=C_{y}[q(\psi) \chi-\varphi], \\
z=\chi
\end{array}\right.
$$

Here, $\psi$ is the poloidal flux, defined as

$$
\psi=\frac{1}{2 \pi} \int_{V} \mathbf{B} \cdot \nabla \theta \mathrm{d}^{3} x
$$

where $V$ is the volume enclosed by a magnetic surface, $\chi$ is the straight-field-line poloidal angle, defined in terms of the geometrical poloidal angle $\theta$ as

$$
\chi=2 \pi \int_{0}^{\theta} \frac{\mathbf{B} \cdot \nabla \varphi}{\mathbf{B} \cdot \nabla \theta^{\prime}} \mathrm{d} \theta^{\prime} / \oint \frac{\mathbf{B} \cdot \nabla \varphi}{\mathbf{B} \cdot \nabla \theta^{\prime}} \mathrm{d} \theta^{\prime},
$$

$\varphi$ is the toroidal angle, and $q$ the safety factor. The magnetic field $\mathbf{B}$ can be written with respect to either $(\psi, \chi, \varphi)$ or $(x, y, z)$ as follows:

$$
\mathbf{B}=B \mathbf{b}=F \nabla \varphi+\frac{1}{2 \pi} \nabla \varphi \times \nabla \psi=\mathcal{C}(\nabla x \times \nabla y),
$$

where $F(\psi)=R B_{\varphi}$, with $B_{\varphi}$ the toroidal component of the magnetic field, and

$$
\mathcal{C}=\frac{1}{2 \pi\left(d C_{x} / d \psi\right) C_{y}}
$$

In the GENE coordinate system, the $x$ coordinate defines the radial direction, $y$ is called the binormal direction, while $z$ parametrizes the position along a field line and therefore is referred to as the "parallel" direction. The exact definition of the radial coordinate $x$ depends on the particular geometry used. One defines $x_{0}$ as a reference position and considers a radial simulation domain $L_{x}$ centered around $x_{0}$. The constant $C_{y}$ appearing in the definition of the $y$-direction (Eq. A1) is chosen as $C_{y}=x_{0} / q_{0}$ in order to establish $y$ as a length rather than an angle-like quantity. Here $q_{0}=q\left(x_{0}\right)$ indicates the local safety factor evaluated at the reference position $x=x_{0}$. In $(x, y, z)$ coordinates, axisymmetry translates to invariance with respect to $y$, so that linear fluctuation eigenmodes have an exact wave-number $k_{y}$ with respect to $y$. The relation between $k_{y}$ and the toroidal mode number $n$ being:

$$
n=k_{y} C_{y} \quad k_{y}=\frac{n}{C_{y}}=n \frac{q_{0}}{x_{0}}
$$


The binormal direction $y$ is thus directly implemented in Fourier space.

[1] X. Garbet, Y. Idomura, L. Villard, and T. Watanabe, Nuclear Fusion 50, 043002 (2010).

[2] J. A. Krommes, Physica Scripta 2010, 014035 (2010).

[3] F. Jenko, W. Dorland, M. Kotschenreuther, and B. N. Rogers, Physics of Plasmas 7, 1904 (2000).

[4] T. Görler, X. Lapillonne, S. Brunner, T. Dannert, F. Jenko, F. Merz, and D. Told, Journal of Computational Physics 230, 7053 (2011), ISSN 0021-9991.

[5] A. E. White, W. A. Peebles, T. L. Rhodes, C. H. Holland, G. Wang, L. Schmitz, T. A. Carter, J. C. Hillesheim, E. J. Doyle, L. Zeng, et al., Physics of Plasmas 17, 056103 (2010).

[6] A. J. Creely, N. T. Howard, P. Rodriguez-Fernandez, N. Cao, A. E. Hubbard, J. W. Hughes, J. E. Rice, A. E. White, J. Candy, G. M. Staebler, et al., Physics of Plasmas 24, 056104 (2017).

[7] C. Lechte, G. D. Conway, T. Görler, C. Tröster-Schmid, and the ASDEX Upgrade Team, Plasma Physics and Controlled Fusion 59, 075006 (2017).

[8] S. J. Freethy, T. Görler, A. J. Creely, G. D. Conway, S. S. Denk, T. Happel, C. Koenen, P. Hennequin, A. E. White, and A. U. Team, Physics of Plasmas 25, 055903 (2018).

[9] S. Ku, C. S. Chang, and P. H. Diamond, Nuclear Fusion 49, 115021 (2009).

[10] S. Ku, C. S. Chang, R. Hager, J. M. Kwon, and S. E. Parker, Journal of Computational Physics 315, 467 (2016).

[11] S. Ku, C. S. Chang, R. Hager, R. M. Churchill, G. R. Tynan, I. Cziegler, M. Greenwald, J. Hughes, S. E. Parker, M. F. Adams, et al., Physics of Plasmas 25, 056107 (2018).

[12] X. Lapillonne, B. F. McMillan, T. Görler, S. Brunner, T. Dannert, F. Jenko, F. Merz, and L. Villard, Physics of Plasmas 17, 112321 (2010).

[13] R. Bravenec, J. Citrin, J. Candy, P. Mantica, T. Görler, and J. contributors, Plasma Physics and Controlled Fusion 58, 125018 (2016).

[14] T. Görler, N. Tronko, W. A. Hornsby, A. Bottino, R. Kleiber, C. Norscini, V. Grandgirard, F. Jenko, and E. Sonnendrücker, Physics of Plasmas 23, 072503 (2016).

[15] G. Dif-Pradalier, P. H. Diamond, V. Grandgirard, Y. Sarazin, J. Abiteboul, X. Garbet, 
P. Ghendrih, A. Strugarek, S. Ku, and C. S. Chang, Phys. Rev. E 82, 025401 (2010).

[16] I. Holod and Z. Lin, Physics of Plasmas 20, 032309 (2013).

[17] K. Kim, C. S. Chang, J. Seo, S. Ku, and W. Choe, Physics of Plasmas 24, 012306 (2017).

[18] R. Hager, J. Lang, C. S. Chang, S. Ku, Y. Chen, S. E. Parker, and M. F. Adams, Physics of Plasmas 24, 054508 (2017).

[19] T. Tran, K. Appert, M. Fivaz, G. Jost, J. Vaclavik, and L. Villard, Theory of fusion plasmas, in: Int. Workshop, Editrice Compositori, Societá italiana di Fisica, Bologna p. 45 (1999).

S. Jolliet, A. Bottino, P. Angelino, R. Hatzky, T. Tran, B. Mcmillan, O. Sauter, K. Appert, Y. Idomura, and L. Villard, Computer Physics Communications 177, 409 (2007).

[20] A. J. Brizard and T. S. Hahm, Rev. Mod. Phys. 79, 421 (2007).

[21] See http://genecode.org.

[22] See http://hbps.pppl.gov.

[23] W. Lee, Journal of Computational Physics 72, 243 (1987), ISSN 0021-9991.

[24] S. Parker, C. Kim, and Y. Chen, Physics of Plasmas 6 (1999).

[25] N. Tronko, A. Bottino, T. Görler, E. Sonnendrücker, D. Told, and L. Villard, Physics of Plasmas 24, 056115 (2017).

[26] J. Dominski, B. F. McMillan, S. Brunner, G. Merlo, T.-M. Tran, and L. Villard, Physics of Plasmas 24, 022308 (2017).

[27] E. Lanti, J. Dominski, S. Brunner, B. F. McMillan, and L. Villard, Journal of Physics: Conference Series 775, 012006 (2016).

[28] A. Burckel, O. Sauter, C. Angioni, J. Candy, E. Fable, and X. Lapillonne, Journal of Physics: Conference Series 260, 012006 (2010).

[29] A. M. Dimits, G. Bateman, M. A. Beer, B. I. Cohen, W. Dorland, G. W. Hammett, C. Kim, J. E. Kinsey, M. Kotschenreuther, A. H. Kritz, et al., Physics of Plasmas 7, 969 (2000).

[30] G. Merlo, O. Sauter, S. Brunner, A. Burckel, Y. Camenen, F. J. Casson, W. Dorland, E. Fable, T. Görler, F. Jenko, et al., Physics of Plasmas 23, 032104 (2016).

[31] B. F. McMillan, X. Lapillonne, S. Brunner, L. Villard, S. Jolliet, A. Bottino, T. Görler, and F. Jenko, Phys. Rev. Lett. 105, 155001 (2010).

[32] G. Merlo, J. Dominski, A. Bhattacharjee, C. S. Chang, F. Jenko, S. Ku, E. Lanti, and S. Parker, Cross-verification of the global gyrokinetic codes GENE and XGC, Zenodo (2018), URL http://dx.doi.org/10.5281/zenodo.898212. 